PROCEEDINGS OF THE

AMERICAN MATHEMATICAL SOCIETY

Volume 131, Number 6, Pages 1857-1866

S 0002-9939(02)06729-1

Article electronically published on October 1, 2002

\title{
A GLOBAL COMPACTNESS RESULT FOR SINGULAR ELLIPTIC PROBLEMS INVOLVING CRITICAL SOBOLEV EXPONENT
}

\author{
DAOMIN CAO AND SHUANGJIE PENG
}

(Communicated by David S. Tartakoff)

\begin{abstract}
Let $\Omega \subset R^{N}$ be a bounded domain such that $0 \in \Omega, N \geq 3,2^{*}=$ $\frac{2 N}{N-2}, \lambda \in R, \epsilon \in R$. Let $\left\{u_{n}\right\} \subset H_{0}^{1}(\Omega)$ be a (P.S.) sequence of the functional $E_{\lambda, \epsilon}(u)=\frac{1}{2} \int_{\Omega}\left(|\nabla u|^{2}-\frac{\lambda u^{2}}{|x|^{2}}-\epsilon u^{2}\right)-\frac{1}{2^{*}} \int_{\Omega}|u|^{2^{*}}$. We study the limit behaviour of $u_{n}$ and obtain a global compactness result.
\end{abstract}

\section{INTRODUCTION} $R$.

Let $\Omega$ be a bounded domain in $R^{N}$ such that $0 \in \Omega, N \geq 3,2^{*}=\frac{2 N}{N-2}, \lambda \in R, \epsilon \in$

In recent years, much attention has been paid to the existence of nontrivial solutions to the following problem:

$$
\left(P_{\lambda, \epsilon}\right) \begin{cases}-\Delta u=\frac{\lambda}{|x|^{2}} u+|u|^{2^{*}-2} u+\epsilon u, & x \in \Omega, \\ u=0, & x \in \partial \Omega,\end{cases}
$$

where $0 \leq \lambda<\bar{\lambda}=\left(\frac{N-2}{2}\right)^{2}, \epsilon \in R$. For instance, in [11, by using a variational approach, E. Jannelli proved that if $\lambda \leq \bar{\lambda}-1$, then problem $\left(P_{\lambda, \epsilon}\right)$ has at least one solution $u \in H_{0}^{1}(\Omega)$ when $0<\epsilon<\epsilon_{1}(\lambda)$. If $\bar{\lambda}-1<\lambda<\bar{\lambda}$, then problem $\left(P_{\lambda, \epsilon}\right)$ has at least one solution $u \in H_{0}^{1}(\Omega)$ when $\epsilon_{*}(\lambda)<\epsilon<\epsilon_{1}(\lambda)$, where $\epsilon_{1}(\lambda), \epsilon_{*}(\lambda)$ are some positive constants depending on $\lambda$.

Let

$$
E_{\lambda, \epsilon}(u)=\frac{1}{2} \int_{\Omega}\left(|\nabla u|^{2}-\frac{\lambda u^{2}}{|x|^{2}}-\epsilon u^{2}\right)-\frac{1}{2^{*}} \int_{\Omega}|u|^{2^{*}}, \text { for } u \in H_{0}^{1}(\Omega) .
$$

The crucial step in [11] was to overcome the lack of compactness for $E_{\lambda, \epsilon}(u)$. Indeed, the invariance of $H_{0}^{1}-$ norm, $L^{2^{*}}-$ norm and $\int_{\Omega} \frac{\lambda u^{2}}{|x|^{2}}$ with respect to rescaling $u \mapsto$ $u_{r}=r^{\frac{N-2}{2}} u(r(\cdot))$ and the existence of non-trivial entire solution of the limiting

Received by the editors December 2, 2001 and, in revised form, January 31, 2002.

2000 Mathematics Subject Classification. Primary 35J60; Secondary 35 B33.

Key words and phrases. Palais-Smale sequence, compactness, Sobolev and Hardy critical exponents.

The first author was supported by Special Funds For Major States Basic Research Projects of China (G1999075107) and Knowledge Innovation Funds of CAS in China.

The second author was supported by Knowledge Innovation Funds of CAS in China.

(C)2002 American Mathematical Society 
problem (see [4], 10, 12] and [14])

$$
\begin{cases}-\Delta u=\frac{\lambda}{|x|^{2}} u+|u|^{2^{*}-2} u, & x \in R^{N}, \\ u \rightarrow 0, & |x| \rightarrow \infty,\end{cases}
$$

result in the failure of the classical Palais-Smale (P.S. for short) condition for $E_{\lambda, \epsilon}$ on $H_{0}^{1}(\Omega)$. However a local (P.S.) condition can be established. Indeed, let $|u|_{p}^{p}=$ $\int_{\Omega}|u|^{p}$ for $p \in(1, \infty)$ and

$$
S_{\lambda}:=\inf \left\{\left.\int_{R^{N}}\left(|\nabla u|^{2}-\frac{\lambda u^{2}}{|x|^{2}}\right)\left|u \in H_{0}^{1}\left(R^{N}\right),\right| u\right|_{2^{*}}=1\right\} .
$$

Suppose $\left\{u_{m}\right\} \subset H_{0}^{1}(\Omega)$ is a sequence such that $E_{\lambda, \epsilon}\left(u_{m}\right) \leq c<\frac{1}{N} S_{\lambda}^{\frac{N}{2}}, D E_{\lambda, \epsilon}\left(u_{m}\right)$ $\rightarrow 0$ strongly in $H^{-1}(\Omega)=\left(H_{0}^{1}(\Omega)\right)^{*}$; then $\left\{u_{m}\right\}$ contains a strongly convergent subsequence. Using this local (P.S.) condition, E.Jannelli was able to obtain the existence of one nontrivial solution in [1]. For earlier work, see [3].

As indicated above it is always very important to understand the convergence of the (P.S.) sequence in using variational methods. When $\lambda=0$, M.Struwe [14] gives a complete description of all energy levels $c$ of $E_{\lambda, \epsilon}$ on which the $(P . S .)_{c}$ sequence is not compact, and he obtains the well known global compactness theorem, with which J.M.Coron [6] and W.-Y. Ding [7] obtained the positive critical points of $E_{0,0}$ with some non-starshaped domains. In [16] S.Yan generalizes this global compactness result to the case of $p$-Laplacian successfully. Very recently, Adimurthi and M.Struwe in [1] have studied the convergence of (P.S.) sequences of the energy functional associated with a semilinear elliptic problem on a bounded domain in $R^{2}$ with critical nonlinearity $f(s)$ growing like $\exp \left(4 \pi s^{2}\right)$ as $s \rightarrow \infty$.

As can easily be seen, problem $\left(P_{\lambda, \epsilon}\right)$ is the general case of problem $\left(P_{0, \epsilon}\right)$. So it is very interesting and important to see whether a global compactness result similar to that of problem $\left(P_{0, \epsilon}\right)$ still exists or not. In this paper we investigate this problem and obtain an affirmative answer.

Our proof is based on rescaling arguments. Such methods have been repeatedly used to extract convergent subsequence from families of solutions or minimizing sequence to nonlinear variational problems; see [13, [14, and [16]).

In the following, let $D^{1,2}\left(R^{N}\right)$ be the completion of $C_{0}^{\infty}\left(R^{N}\right)$ with respect to the inner product $(u, v)=\int_{R^{N}} \nabla u \cdot \nabla v, S_{0}=\inf \left\{\left.\int_{R^{N}}|\nabla u|^{2}\left|u \in H_{0}^{1}\left(R^{N}\right),\right| u\right|_{2^{*}}=1\right\}$. Let $B(x, r)$ denote the ball centered at $x$ with radius $r$. For simplicity, we use the same notation $\tilde{\Omega}_{m}$ and $\tilde{v}_{m}$ in different situations.

Remark 1.1. (i) By Proposition 8.1 in [4, the solutions of (1.2) are in one-to-one correspondence to solutions of the following type of problem:

$$
-\operatorname{div}\left(|x|^{-2 a} \nabla w\right)=|x|^{b 2^{*}} w^{2^{*}-1}, w \geq 0 \text { in } R^{N},
$$

where $a$ and $b$ are constants depending on $\lambda$. From Theorem B in [5], any solution of the above equations is radially symmetric with respect to the origin, that is, $u(x)$ depends on $|x|$ only. So according to the theory of ordinary differential equations, the solution of (1.2) is unique (up to a dilation). Using the results in [4, we can deduce that when $0 \leq \lambda<\bar{\lambda}$, the solutions of (1.3) are of the following:

$$
\begin{gathered}
u(x)=\varepsilon^{\frac{N-2}{2}} U(\varepsilon x), \quad \varepsilon>0, \\
U(x)=C(\lambda, \bar{\lambda})|x|^{-\sqrt{\lambda}+\sqrt{\bar{\lambda}-\lambda}}\left(1+|x|^{\frac{4 \sqrt{\lambda-\lambda}}{N-2}}\right),
\end{gathered}
$$


where $C(\bar{\lambda}, \lambda)$ is a constant depending only on $\lambda$ and $\bar{\lambda}$. So $S_{\lambda}$ can be attained in $R^{N}$ when $0 \leq \lambda<\bar{\lambda}$.

(ii) $S_{\lambda}<S_{0}$ when $0<\lambda<\bar{\lambda}$. While $\lambda<0$, we can prove that $S_{\lambda}=S_{0}$ and $S_{\lambda}$ cannot be attained, even though in $R^{N}$.

\section{Global compactness}

To state the main result, it is convenient to introduce the "problem at infinity". The first one is

$\left(P_{0}^{\infty}\right) \quad-\Delta v=|v|^{2^{*}-2} v, \quad v \in D^{1,2}\left(R^{N}\right)$.

Moreover, for $0 \leq \lambda<\bar{\lambda}$, the problem "at infinity" is given by

$\left(P_{\lambda}^{\infty}\right) \quad-\Delta v=\frac{\lambda v}{|x|^{2}}+|v|^{2^{*}-2} v, \quad v \in D^{1,2}\left(R^{N}\right)$.

To unify the notations we shall refer to the solutions of problems at infinity as critical points of the following family of functionals:

$$
F_{\lambda}^{\infty}(u)=\frac{1}{2} \int_{R^{N}}\left(|\nabla u|^{2}-\frac{\lambda u^{2}}{|x|^{2}}\right)-\frac{1}{2^{*}} \int_{R^{N}}|u|^{2^{*}}
$$

and in the cases of $\left(P_{0}^{\infty}\right)$ and $\left(P_{\lambda}^{\infty}\right)$, the respective energy functionals are $F_{0}^{\infty}(u)$ and $F_{\lambda}^{\infty}(u)$.

Theorem 2.1. Let $N \geq 3,0 \leq \lambda<\bar{\lambda}, \epsilon \in R$, and suppose that $\left\{u_{m}\right\} \subset H_{0}^{1}(\Omega)$ satisfies $E_{\lambda, \epsilon}\left(u_{m}\right) \leq c, D E_{\lambda, \epsilon}\left(u_{m}\right) \rightarrow 0$ strongly in $H^{-1}(\Omega)$ as $m \rightarrow \infty$. Then there exist a critical point $u^{0}$ of $E_{\lambda, \epsilon}, k$ sequences of positive numbers $\left\{r_{m}^{j}\right\}_{m}(1 \leq j \leq k)$, $l$ sequences of positive numbers $\left\{k_{m}^{j}\right\}_{m}(1 \leq j \leq l)$ and $l$ sequences of points $\left\{x_{m}^{j}\right\}_{m}(1 \leq j \leq l)$ in $\Omega$ which converge to $x_{0}^{j} \in \bar{\Omega}$, such that up to a subsequence,

$$
\text { (i) } u_{m}=u^{0}+\sum_{j=1}^{k}\left(r_{m}^{j}\right)^{\frac{N-2}{2}} v_{1}^{j}\left(r_{m}^{j} x\right)+\sum_{j=1}^{l}\left(k_{m}^{j}\right)^{\frac{N-2}{2}} v_{0}^{j}\left(k_{m}^{j}\left(x-x_{m}^{j}\right)\right)+\omega_{m},
$$

where $\left\|\omega_{m}\right\|_{H_{0}^{1}(\Omega)} \rightarrow 0, r_{m}^{j} \rightarrow \infty, k_{m}^{j} \rightarrow \infty, v_{0}^{j}$ solves $\left(P_{0}^{\infty}\right)$, and $v_{1}^{j}$ solves $\left(P_{\lambda}^{\infty}\right)$.

$$
\text { (ii) } E_{\lambda, \epsilon}\left(u_{m}\right) \rightarrow E_{\lambda, \epsilon}\left(u^{0}\right)+\sum_{j=1}^{l} F_{0}^{\infty}\left(v_{0}^{j}\right)+\sum_{j=1}^{k} F_{\lambda}^{\infty}\left(v_{1}^{j}\right) .
$$

To prove this theorem, let us first recall some known results and establish some preliminary lemmas. The following lemma can be found in [9].

Lemma 2.2 (Hardy inequality). If $u \in H_{0}^{1}(\Omega)$, then $\frac{u}{|x|^{2}} \in L^{2}(\Omega)$ and

$$
\int_{\Omega} \frac{u^{2}}{|x|^{2}} \leq \frac{1}{\bar{\lambda}} \int_{\Omega}|\nabla u|^{2}
$$

moreover $\bar{\lambda}$ is optimal.

Lemma 2.3. Let $\left\{u_{m}\right\} \subset H_{0}^{1}(\Omega), u_{m} \rightarrow u$ weakly in $H_{0}^{1}(\Omega)$. Then

(i) $\int_{\Omega}\left|u_{m}\right|^{2^{*}}=\int_{\Omega}\left|u_{m}-u\right|^{2^{*}}+\int_{\Omega}|u|^{2^{*}}+o(1)$,

(ii) $\int_{\Omega}\left|\nabla u_{m}\right|^{2}=\int_{\Omega}\left|\nabla\left(u_{m}-u\right)\right|^{2}+\int_{\Omega}|\nabla u|^{2}+o(1)$,

(iii) $\int_{\Omega} \frac{\left|u_{m}\right|^{2}}{|x|^{2}}=\int_{\Omega} \frac{\left|u_{m}-u\right|^{2}}{|x|^{2}}+\int_{\Omega} \frac{|u|^{2}}{|x|^{2}}+o(1)$. 
Proof. (i) and (ii) are the well known results of Brezis-Lieb in [2]. (iii) can be easily proved by Vitali's theorem and we omit the detail.

Lemma 2.4. Let $\left\{v_{m}\right\} \subset H_{0}^{1}(\Omega)$ be a (P.S.) sequence for $E_{\lambda, 0}$ at level $\beta$, that is, $E_{\lambda, 0}\left(v_{m}\right) \rightarrow \beta$ as $m \rightarrow \infty$, and assume that $v_{m}$ converges weakly but not strongly to zero in $H_{0}^{1}(\Omega)$. Then either

(i) there exists a sequence of positive numbers $\left\{r_{m}\right\}$, such that up to a subsequence,

$$
w_{m}(x)=v_{m}(x)-r_{m}^{\frac{N-2}{2}} V_{1}\left(r_{m} x\right)+o(1) \quad(x \in \Omega)
$$

is a (P.S.) sequence for $E_{\lambda, 0}$ in $H_{0}^{1}(\Omega)$ at level $\beta-F_{\lambda}^{\infty}\left(V_{1}\right)$; moreover, $w_{m} \rightarrow 0$ weakly in $H_{0}^{1}(\Omega)$,

or

(ii) there exists a sequence of positive numbers $\left\{k_{m}\right\}$ and a sequence of points $\left\{y_{m}\right\} \subset \Omega$ satisfying $y_{m} \rightarrow x_{0} \in \bar{\Omega}$, such that up to a subsequence,

$$
w_{m}(x)=v_{m}(x)-k_{m}^{\frac{N-2}{2}} V_{0}\left(k_{m}\left(x-y_{m}\right)\right)+o(1) \quad(x \in \Omega)
$$

is a (P.S.) sequence for $E_{\lambda, 0}$ in $H_{0}^{1}(\Omega)$ at level $\beta-F_{0}^{\infty}\left(V_{0}\right)$. Moreover, $w_{m} \rightarrow 0$ weakly in $H_{0}^{1}(\Omega), k_{m} \operatorname{dist}\left(x_{m}, \partial \Omega\right) \rightarrow \infty$, where $V_{0}$ and $V_{1}$ solve $\left(P_{0}^{\infty}\right)$ and $\left(P_{\lambda}^{\infty}\right)$ respectively, o(1) $\rightarrow 0$ in $D^{1,2}\left(R^{N}\right)$ as $m \rightarrow \infty$.

Proof. If $E_{\lambda, 0}\left(v_{m}\right) \rightarrow \beta<\frac{1}{N} S_{\lambda}^{\frac{N}{2}}$, then sequence $\left\{v_{m}\right\}$ is strongly relatively compact and hence $v_{m} \rightarrow 0, \beta=0$. Therefore we may assume that $E_{\lambda, 0}\left(v_{m}\right) \rightarrow \beta \geq \frac{1}{N} S_{\lambda}^{\frac{N}{2}}$. Moreover, from $D E_{\lambda, 0}\left(v_{m}\right) \rightarrow 0$, we also have

$$
\frac{1}{N} \int_{\Omega}\left(\left|\nabla v_{m}\right|^{2}-\frac{\lambda v_{m}^{2}}{\left|x^{2}\right|}\right)=E_{\lambda, 0}\left(v_{m}\right)-\frac{1}{2}\left\langle D E_{\lambda, 0}\left(v_{m}\right), v_{m}\right\rangle \rightarrow \beta \geq \frac{1}{N} S_{\lambda}^{\frac{N}{2}} .
$$

By Hardy inequality, we have for $m$ large enough

$$
S_{\lambda}^{\frac{N}{2}} \leq N \beta \leq \int_{\Omega}\left|\nabla v_{m}\right|^{2} \leq \frac{N \beta}{1-\frac{\lambda}{\lambda}}
$$

and hence there are positive constants $c_{i}(i=1,2)$ such that

$$
c_{1} \leq \int_{\Omega}\left|\nabla v_{m}\right|^{2} \leq c_{2}, \quad \forall m .
$$

Let $\delta>0$ small (will be determined later) such that

$$
\limsup _{m \rightarrow \infty} \int_{\Omega}\left|\nabla v_{m}\right|^{2}>\delta .
$$

Up to a subsequence, choose minimal $\frac{1}{r_{m}}>0$ such that $\int_{B\left(0, \frac{1}{r_{m}}\right)}\left|\nabla v_{m}\right|^{2}=\delta$. Define $\tilde{v}_{m}:=r_{m}^{\frac{2-N}{2}} v_{m}\left(\frac{x}{r_{m}}\right) ;$ then $\int_{B(0,1)}\left|\nabla \tilde{v}_{m}\right|^{2}=\delta$.

Let us point out that, thanks to (2.3) and (2.4), the sequence $\left\{r_{m}\right\}$ is bounded away from zero.

Denote $\tilde{\Omega}_{m}:=\left\{x \in R^{N} \mid \frac{x}{r_{m}} \in \Omega\right\}$; then $\tilde{v}_{m} \in H_{0}^{1}\left(\tilde{\Omega}_{m}\right) \subset D^{1,2}\left(R^{N}\right)$. Moreover, $\left\|\tilde{v}_{m}\right\|_{D^{1,2}\left(R^{N}\right)}^{2}=\left\|v_{m}\right\|_{D^{1,2}\left(R^{N}\right)}^{2} \rightarrow N \beta<\infty$. Up to a subsequence there exists $V_{1} \in D^{1,2}\left(R^{N}\right)$ satisfying $\tilde{v}_{m} \rightarrow V_{1}$ weakly in $D^{1,2}\left(R^{N}\right), \tilde{v}_{m} \rightarrow V_{1}$ a.e. in $R^{N}$. We have either $V_{1} \not \equiv 0$ or $V_{1} \equiv 0$. 
(I): Assume $V_{1} \not \equiv 0$. Since $v_{m} \rightarrow 0$ weakly in $H_{0}^{1}(\Omega)$, we have $r_{m} \rightarrow \infty, \tilde{\Omega}_{m} \rightarrow$ $R^{N}$. For this case we claim that $V_{1}$ satisfies $\left(P_{\lambda}^{\infty}\right)$ and the sequence $w_{m}(x):=$ $\tilde{v}_{m}(x)-r_{m}^{\frac{N-2}{2}} V_{1}\left(r_{m} x\right)$ is a (P.S.) sequence for $E_{\lambda, 0}$ at level $\beta-F_{\lambda}^{\infty}\left(V_{1}\right)$.

Indeed, let us fix a ball $B(0, r)$ and a test function $\psi \in C_{0}^{\infty}(B(0, r))$ and remark that for sufficiently large $m, B(0, r) \subset \tilde{\Omega}_{m}$. So

$$
\begin{aligned}
& \left\langle D F_{\lambda}^{\infty}\left(V_{1}\right), \psi\right\rangle \\
& =\int_{B(0, r)} \nabla V_{1} \cdot \nabla \psi-\int_{B(0, r)} \frac{\lambda V_{1} \psi}{|x|^{2}}-\int_{B(0, r)}\left|V_{1}\right|^{2^{*}-2} V_{1} \psi \\
& =\int_{\tilde{\Omega}_{m}} \nabla \tilde{v}_{m} \cdot \nabla \psi-\int_{\tilde{\Omega}_{m}} \frac{\lambda \tilde{v}_{m} \psi}{|x|^{2}}-\int_{\tilde{\Omega}_{m}}\left|\tilde{v}_{m}\right|^{2^{*}-2} \tilde{v}_{m} \psi+o(1) \\
& =\int_{\Omega} \nabla v_{m} \cdot \nabla \bar{\psi}_{m}-\int_{\Omega} \frac{\lambda v_{m} \bar{\psi}_{m}}{|x|^{2}}-\int_{\Omega}\left|v_{m}\right|^{2^{*}-2} v_{m} \bar{\psi}_{m}+o(1) \\
& =\left\langle D E_{\lambda, 0}\left(v_{m}\right), \bar{\psi}_{m}\right\rangle+o(1) \quad \text { (by the (P.S.) condition) }
\end{aligned}
$$

where $\bar{\psi}_{m}(x)=r_{m}^{\frac{N-2}{2}} \psi\left(r_{m} x\right)$. Thus $V_{1}$ solves $\left(P_{\lambda}^{\infty}\right)$.

Let $\varphi \in C_{0}^{\infty}\left(R^{N}\right)$ satisfying $0 \leq \varphi \leq 1,|\nabla \varphi| \stackrel{\leq}{\leq}$ in $R^{N}, \varphi \equiv 1$, in $B(0,1), \varphi \equiv 0$ outside $B(0,2)$, and let

$$
w_{m}(x)=v_{m}(x)-r_{m}^{\frac{N-2}{2}} V_{1}\left(r_{m} x\right) \varphi\left(\bar{r}_{m} x\right) \in H_{0}^{1}(\Omega)
$$

where the sequence $\left\{\bar{r}_{m}\right\}$ is chosen such that $\tilde{r}_{m}:=\frac{r_{m}}{\bar{r}_{m}} \rightarrow \infty$ while $\bar{r}_{m} \operatorname{dist}\left(0, \partial \tilde{\Omega}_{m}\right)$ $\rightarrow \infty$ as $m \rightarrow \infty$, that is, $\tilde{w}_{m}(x)=\tilde{v}_{m}(x)-V_{1}(x) \varphi\left(\frac{x}{\tilde{r}_{m}}\right)$.

Set $\varphi_{m}(x)=\varphi\left(\frac{x}{\tilde{r}_{m}}\right)$. Note that $\left|\nabla V_{1}\right| \in L^{2}\left(R^{N}\right)$, so as $m \rightarrow \infty$,

$$
\begin{aligned}
& \int_{R^{N}}\left|\nabla V_{1}\left(\varphi_{m}-1\right)\right|^{2} \\
& \leq 2 \int_{R^{N}}\left|\nabla V_{1}\right|^{2}\left(\varphi_{m}-1\right)^{2}+2 \int_{R^{N}}\left|V_{1}\right|^{2}\left|\nabla\left(\varphi_{m}-1\right)^{2}\right| \\
& \leq 2 \int_{R^{N} \backslash B\left(0, r_{m}\right)}\left|\nabla V_{1}\right|^{2}+8 \tilde{r}_{m}^{-2} \int_{B\left(0,2 \tilde{r}_{m}\right) \backslash B\left(0, \tilde{r}_{m}\right)}\left|V_{1}\right|^{2} \\
& \leq 2 \int_{R^{N} \backslash B\left(0, r_{m}\right)}\left|\nabla V_{1}\right|^{2}+8\left(\int_{B\left(0,2 \tilde{r}_{m}\right) \backslash B\left(0, \tilde{r}_{m}\right)}\left|V_{1}\right|^{2^{*}}\right)^{\frac{2}{2^{*}}} \\
& =o(1) .
\end{aligned}
$$

Thus we have $\tilde{w}_{m}=\tilde{v}_{m}-V_{1}+o(1)$, where $o(1) \rightarrow 0$ in $D^{1,2}\left(R^{N}\right)$. So by Lemma 2.3 and the invariance of dilation, we have for $m$ large

$$
\begin{gathered}
E_{\lambda, 0}\left(w_{m}\right)=E_{\lambda, 0}\left(\tilde{v}_{m}\right)-F_{\lambda}^{\infty}\left(V_{1}\right)+o(1)=\beta-F_{\lambda}^{\infty}\left(V_{1}\right)+o(1), \\
\left\|D E_{\lambda, 0}\left(w_{m}\right)\right\|_{H^{-1}(\Omega)}=o(1) .
\end{gathered}
$$

Also, it is obvious that $w_{m} \rightarrow 0$ weakly in $H_{0}^{1}(\Omega)$. 
(II): Assume $V_{1} \equiv 0$. Let $h \in C_{0}^{\infty}(B(0,1))$; we have

$$
\begin{aligned}
& \int_{R^{N}}\left|\nabla\left(\tilde{v}_{m} h\right)\right|^{2} \\
& =\int_{R^{N}} \nabla \tilde{v}_{m} \cdot \nabla\left(h^{2} \tilde{v}_{m}\right)+\int_{R^{N}} \tilde{v}_{m}^{2}|\nabla h|^{2} \\
& =\int_{R^{N}} \nabla \tilde{v}_{m} \cdot \nabla\left(h^{2} \tilde{v}_{m}\right)+o(1) \\
& =\left\langle D E_{\lambda, 0}\left(\tilde{v}_{m}\right), h^{2} \tilde{v}_{m}\right\rangle+\int_{R^{N}} \frac{\lambda h^{2} \tilde{v}_{m}}{|x|^{2}}+\int_{R^{N}}\left|\tilde{v}_{m}\right|^{2^{*}} h^{2}+o(1) \\
& \leq \frac{4 \lambda}{(N-2)^{2}} \int_{R^{N}}\left|\nabla\left(\tilde{v}_{m} h\right)\right|^{2}+S_{0}^{-1}\left(\int_{B(0,1)}\left|\tilde{v}_{m}\right|^{2^{*}}\right)^{\frac{N}{2}} \int_{R^{N}}\left|\nabla\left(\tilde{v}_{m} h\right)\right|^{2}+o(1) .
\end{aligned}
$$

Choose $\delta$ suitably small. From the above inequality and the fact $0 \leq \lambda<\frac{(N-2)^{2}}{4}$, we can find an $a \in(0,1)$ such that

$$
\int_{B(0, a)}\left|\nabla \tilde{v}_{m}\right|^{2} \rightarrow 0
$$

For simplicity, substitute $\tilde{v}_{m}$ by $z_{m}$ and $\tilde{\Omega}_{m}$ by $\Omega$ (because of dilation invariance), and without loss of generality, we still suppose that $z_{m}$ satisfies (2.3) and (2.4). Denote

$$
Q_{m}(r)=\sup _{x \in \Omega} \int_{B(x, r)}\left|\nabla z_{m}\right|^{2}
$$

the concentration function of $z_{m}$, choose $x_{m} \in \bar{\Omega}$ and scale

$$
z_{m} \mapsto \tilde{z}_{m}(x):=R_{m}^{\frac{2-N}{2}} z_{m}\left(\frac{x}{R_{m}}+x_{m}\right)
$$

such that

$$
\tilde{Q}_{m}(1)=\sup _{\frac{x}{R_{m}}+x_{m}, x \in R^{N}} \int_{B(x, 1)}\left|\nabla \tilde{z}_{m}\right|^{2}=\int_{B(0,1)}\left|\nabla \tilde{z}_{m}\right|^{2}=\frac{1}{2 L} S_{\lambda}^{\frac{N}{2}},
$$

where $L$ denotes the least number of balls with radius 1 in $R^{N}$ that are needed to cover a ball of radius 2 .

Note that $\left\{R_{m}\right\}$ is obviously bounded away from zero. Setting $\tilde{\Omega}_{m}:=\{x \in$ $\left.R^{N} \mid \frac{x}{R_{m}}+x_{m} \in \Omega\right\}$, we may regard $\tilde{z}_{m} \in D^{1,2}\left(R^{N}\right)$, moreover, $\left\{\tilde{z}_{m}\right\}$ is bounded uniformly in $D^{1,2}\left(R^{N}\right)$. Thus up to a subsequence,

$$
\tilde{z}_{m} \rightarrow V_{0} \text { weakly in } D^{1,2}\left(R^{N}\right) \text {. }
$$

We are going to prove that the convergence actually holds in the strong $H_{l o c}^{1}\left(R^{N}\right)$ sense. To do this, following the analogous argument in [14], for any $x \in R^{N}$, we can find $\rho \in[1,2]$ such that the solution $\phi_{m}$ of the Dirichlet problems

satisfies

$$
\begin{cases}-\Delta \phi=0, & x \in B(x, 3) \backslash B(x, \rho), \\ \left.\phi\right|_{\partial B(x, \rho)}=\tilde{z}_{m}-V_{0}, & \left.\phi\right|_{\partial B(x, 3)}=0,\end{cases}
$$

$$
\phi_{m} \rightarrow 0 \text { in } H^{1}(B(x, 3) \backslash B(x, \rho)) .
$$

Let

$$
\varphi_{m}= \begin{cases}\tilde{z}_{m}-V_{0}, & x \in B(x, \rho) \\ \phi_{m}, & x \in B(x, 3) \backslash B(x, \rho) .\end{cases}
$$


It follows that

$$
\varphi_{m}=\tilde{\varphi}_{m}+o(1) \in H_{0}^{1}\left(\tilde{\Omega}_{m}\right)+D^{1,2}\left(R^{N}\right),
$$

where $\tilde{\varphi}_{m} \in H_{0}^{1}\left(\tilde{\Omega}_{m}\right)$ and $o(1) \rightarrow 0$ in $D^{1,2}\left(R^{N}\right)$ as $m \rightarrow \infty$ (see for instance [14). Hence, scaling back the $\varphi_{m}$ 's,

$$
\bar{\varphi}_{m}(x):=R_{m}^{\frac{N-2}{2}} \varphi_{m}\left(R_{m}\left(x-x_{m}\right)\right),
$$

we have, taking into account of (2.9), (2.10) and (2.11),

$$
\left\|\nabla \bar{\varphi}_{m}\right\|_{L^{2}(\Omega)}^{2}=\left\|\varphi_{m}\right\|_{D^{1,2}\left(R^{N}\right)}^{2}+o(1)=\left\|\tilde{z}_{m}-V_{0}\right\|_{D^{1,2}(B(x, \rho))}^{2}+o(1) .
$$

By scale invariance, (2.11) and the fact $\left\{z_{m}\right\}$ is a (P.S.) sequence for $E_{\lambda, 0}$,

$$
\left\langle D E_{m}\left(\tilde{z}_{m}\right), \varphi_{m}\right\rangle=\left\langle D E_{\lambda, 0}\left(z_{m}\right), \bar{\varphi}_{m}\right\rangle+o(1)=o(1)
$$

where

$$
E_{m}\left(\tilde{z}_{m}\right):=\frac{1}{2} \int_{\tilde{\Omega}_{m}}\left(\left|\nabla \tilde{z}_{m}\right|^{2}-\frac{\lambda \tilde{z}_{m}^{2}}{\left|x+R_{m} x_{m}\right|^{2}}\right)-\frac{1}{2^{*}} \int_{\tilde{\Omega}_{m}}\left|\tilde{z}_{m}\right|^{2^{*}} .
$$

Therefore, from the definitions of $E_{m}, \varphi_{m}$ and (2.9), we infer by using arguments similar to those in [14]

$$
\begin{aligned}
o(1)= & \int_{\tilde{\Omega}_{m} \cap B(x, \rho)} \nabla \tilde{z}_{m} \cdot \nabla\left(\tilde{z}_{m}-V_{0}\right)-\int_{\tilde{\Omega}_{m} \cap B(x, \rho)} \frac{\lambda \tilde{z}_{m}\left(\tilde{z}_{m}-V_{0}\right)}{\left|x+R_{m} x_{m}\right|^{2}} \\
& -\int_{\tilde{\Omega}_{m} \cap B(x, \rho)}\left|\tilde{z}_{m}\right|^{2^{*}-2} \tilde{z}_{m}\left(\tilde{z}_{m}-V_{0}\right) \\
= & \int_{\tilde{\Omega}_{m} \cap B(x, \rho)}\left|\nabla\left(\tilde{z}_{m}-V_{0}\right)\right|^{2}-\int_{\tilde{\Omega}_{m} \cap B(x, \rho)} \frac{\lambda\left(\tilde{z}_{m}-V_{0}\right)^{2}}{\left|x+R_{m} x_{m}\right|^{2}} \\
& -\int_{\tilde{\Omega}_{m} \cap B(x, \rho)}\left|\tilde{z}_{m}-V_{0}\right|^{2^{*}}+o(1) \\
= & \int_{\tilde{\Omega}_{m}}\left|\nabla \varphi_{m}\right|^{2}-\int_{\tilde{\Omega}_{m}} \frac{\lambda \varphi_{m}^{2}}{\left|x+R_{m} x_{m}\right|^{2}}-\int_{\tilde{\Omega}_{m}}\left|\varphi_{m}\right|^{2^{*}}+o(1) .
\end{aligned}
$$

Moreover, by scale invariance, Sobolev inequality and Hardy inequality,

$$
\begin{aligned}
o(1) & =\int_{\Omega}\left|\nabla \bar{\varphi}_{m}\right|^{2}-\int_{\Omega} \frac{\lambda \bar{\varphi}_{m}^{2}}{|x|^{2}}-\int_{\Omega}\left|\bar{\varphi}_{m}\right|^{2^{*}} \\
& \geq\left(\int_{\Omega}\left|\nabla \bar{\varphi}_{m}\right|^{2}-\int_{\Omega} \frac{\lambda \bar{\varphi}_{m}^{2}}{|x|^{2}}\right)\left(1-\frac{\left\|\nabla \bar{\varphi}_{m}\right\|_{L^{2}(\Omega)}^{2^{*}-2}}{S_{\lambda}^{\frac{2^{*}}{2}}}\right) \\
& \geq c \int_{\Omega}\left|\nabla \bar{\varphi}_{m}\right|^{2}\left(1-\frac{\left\|\tilde{z}_{m}\right\|_{D^{1,2}(B(x, \rho))}^{2^{*}-2}}{S_{\lambda}^{\frac{2^{*}}{2}}}\right),
\end{aligned}
$$

where (2.12) and the convergence of $\tilde{z}_{m}$ to $V_{0}$ have been used to get the last inequality. Recalling (2.7) we have $\left\|\nabla \tilde{z}_{m}\right\|_{L^{2}(B(x, \rho))}^{2} \leq L\left\|\nabla \tilde{z}_{m}\right\|_{L^{2}(B(0,1))}^{2} \leq \frac{1}{2} S_{\lambda}^{\frac{N}{2}}$, so that (2.13) yields $\left\|\nabla \bar{\varphi}_{m}\right\|_{L^{2}(\Omega)} \rightarrow 0$. From (2.12) we obtain $\left\|\tilde{z}_{m}-V_{0}\right\|_{D^{1,2}(B(x, \rho))} \rightarrow 0$. In particular, $\int_{B(0,1)}\left|\nabla V_{0}\right|^{2}=\frac{1}{2 L} S_{\lambda}^{\frac{N}{2}}>0$ and $V_{0} \not \equiv 0$. By $z_{m} \rightarrow 0$ weakly, we also have $R_{m} \rightarrow \infty$ as $m \rightarrow \infty$.

Now using the result of case (I), we have

$$
\tilde{z}_{m}(x)=R_{m}^{\frac{2-N}{2}} \tilde{v}_{m}\left(\frac{x}{R_{m}}+x_{m}\right)=\left(R_{m} r_{m}\right)^{\frac{2-N}{2}} v_{m}\left(\frac{x}{R_{m} r_{m}}+\frac{x_{m}}{r_{m}}\right) .
$$


Define $k_{m}=R_{m} r_{m}, y_{m}=\frac{x_{m}}{r_{m}}$; then $y_{m} \rightarrow y_{0} \in \bar{\Omega}, k_{m}\left|y_{m}\right|=R_{m}\left|x_{m}\right|$. By (2.5) we have $\left|x_{m}\right|>a>0$, so $k_{m}\left|y_{m}\right| \rightarrow+\infty$. Also, by the fact that $\left\{r_{m}\right\}$ is bounded away from zero, $k_{m} \rightarrow+\infty(m \rightarrow \infty)$. Denote $\tilde{\Omega}_{m}=\left\{x \in R^{N} \mid \frac{x}{k_{m}}+y_{m} \in \Omega\right\}, \tilde{v}_{m}=$ $k_{m^{2}}^{\frac{2-N}{2}} v_{m}\left(\frac{x}{k_{m}}+y_{m}\right)$.

Now we distinguish two cases:

(1) $k_{m} \operatorname{dist}\left(y_{m}, \partial \Omega\right) \leq c<\infty$, uniformly. Then after an orthogonal transformation,

$$
\tilde{\Omega}_{m} \rightarrow \tilde{\Omega}_{\infty}=R_{+}^{N}=\left\{x=\left(x_{1}, \ldots, x_{N}\right), x_{1}>0\right\},
$$

(2) $k_{m} \operatorname{dist}\left(y_{m}, \partial \Omega\right) \rightarrow \infty$, in this case $\tilde{\Omega}_{m} \rightarrow \tilde{\Omega}_{\infty}=R^{N}$.

In each case we have for large $m$ and any given $\varphi \in C_{0}^{\infty}\left(\tilde{\Omega}_{\infty}\right), \int_{\tilde{\Omega}_{m}} \frac{\tilde{v}_{m} \varphi}{\left|x+k_{m} y_{m}\right|^{2}}=$ $o(1)$. Hence there holds, as $m \rightarrow \infty$,

$$
\begin{aligned}
& \int_{\tilde{\Omega}_{\infty}}\left(\nabla V_{0} \cdot \nabla \varphi-\left|V_{0}\right|^{2^{*}-2} V_{0} \varphi\right) \\
& =\int_{\tilde{\Omega}_{m}}\left(\nabla \tilde{v}_{m} \cdot \nabla \varphi-\frac{\lambda \tilde{v}_{m} \varphi}{\left|x+k_{m} y_{m}\right|^{2}}-\left|\tilde{v}_{m}\right|^{2^{*}-2} \tilde{v}_{m} \varphi\right)+o(1) \\
& =\int_{\Omega}\left(\nabla v_{m} \cdot \nabla \varphi-\frac{\lambda v_{m} \varphi}{|x|^{2}}-\left|v_{m}\right|^{2^{*}-2} v_{m} \varphi\right)+o(1) \\
& =o(1) .
\end{aligned}
$$

If $\tilde{\Omega}_{\infty}=R_{+}^{N}$, (2.14) implies that $V_{0} \in H_{0}^{1}\left(\tilde{\Omega}_{\infty}\right)$ is a weakly solution of the equation

$$
-\Delta u=u^{2 *-1}, \quad u>0, \quad x \in R_{+}^{N} ; \quad u=0, x \in \partial R_{+}^{N} .
$$

Thus, $V_{0} \equiv 0$, which is impossible. So case $(2)$ is true, that is, $V_{0}$ solves $\left(P_{0}^{\infty}\right)$.

Let

$$
w_{m}(x)=v_{m}(x)-k_{m}^{\frac{N-2}{2}} V_{0}\left(k_{m}\left(x-y_{m}\right)\right) \varphi\left(\bar{k}_{m}\left(x-y_{m}\right)\right) \in H_{0}^{1}(\Omega),
$$

where $\bar{k}_{m}, \varphi$ are defined similarly to case (I). We can also get $\tilde{w}_{m}=\tilde{v}_{m}-V_{0}+o(1)$, where $o(1) \rightarrow 0$ in $D^{1,2}\left(R^{N}\right)$. Using the fact that $k_{m}\left|y_{m}\right| \rightarrow+\infty$, the invariance of scaling and Lemma 2.3 we obtain that $w_{m}(x)=v_{m}(x)-k_{m}^{\frac{N-2}{2}} V_{0}\left(k_{m}\left(x-x_{m}\right)\right)+o(1)$ is still a (P.S.) sequence for $E_{\lambda, 0}$ at level $\beta-F_{0}^{\infty}\left(V_{0}\right)$ and of course it converges weakly to zero.

This concludes the proof of Lemma 2.4.

Proof of Theorem 2.1. By $E_{\lambda, \epsilon}\left(u_{m}\right) \leq c, D E_{\lambda, \epsilon}\left(u_{m}\right) \rightarrow 0$ strongly in $H^{-1}(\Omega)$, we have $u_{m} \rightarrow u^{0}$ weakly in $H_{0}^{1}(\Omega)$ and $u^{0}$ solves problem $\left(P_{\lambda, \epsilon}\right)$. Moreover, setting $v_{m}=u_{m}-u^{0}$, we then have $v_{m} \rightarrow 0$ strongly in $L^{2}(\Omega)$ and by Lemma 2.3 we get

$$
\begin{aligned}
& \int_{\Omega}\left|u_{m}\right|^{2^{*}}=\int_{\Omega}\left|u_{m}-u\right|^{2^{*}}+\int_{\Omega}|u|^{2^{*}}+o(1), \\
& \int_{\Omega}\left|\nabla u_{m}\right|^{2}=\int_{\Omega}\left|\nabla\left(u_{m}-u\right)\right|^{2}+\int_{\Omega}|\nabla u|^{2}+o(1), \\
& \int_{\Omega} \frac{\left|u_{m}\right|^{2}}{|x|^{2}}=\int_{\Omega} \frac{\left|u_{m}-u\right|^{2}}{|x|^{2}}+\int_{\Omega} \frac{|u|^{2}}{|x|^{2}}+o(1) .
\end{aligned}
$$


Hence,

$$
\begin{aligned}
& E_{\lambda, \epsilon}\left(u_{m}\right)=E_{\lambda, \epsilon}\left(u^{0}\right)+E_{\lambda, 0}\left(v_{m}\right)+o(1), \\
& D E_{\lambda, \epsilon}\left(u_{m}\right)=D E_{\lambda, \epsilon}\left(u^{0}\right)+D E_{\lambda, 0}\left(v_{m}\right)+o(1), \\
& D E_{\lambda, 0}\left(v_{m}\right)=o(1) .
\end{aligned}
$$

By applying Lemma2.4 to $\left\{v_{m}\right\}$ recursively, the iteration must stop after finite steps; moreover the last (P.S.) sequence must strongly converge to zero.

Applying Theorem 2.1 we can prove the following:

Corollary 2.5. Suppose $\frac{1}{N} S_{\lambda}^{\frac{N}{2}}<c<\frac{2}{N} S_{\lambda}^{\frac{N}{2}}$ and $c \neq \frac{1}{N} S_{0}^{\frac{N}{2}}$, then functional $E_{\lambda, 0}$ satisfies the $(P . S .)_{c}$ condition.

Proof. Suppose $\left\{u_{m}\right\}$ is a $(P . S .)_{c}$ sequence for $E_{\lambda, 0}(u)$. Notice that for any $u \in$ $D^{1,2}\left(R^{N}\right) \backslash\{0\}$ satisfying $\left(P_{\lambda}^{\infty}\right)(0 \leq \lambda<\bar{\lambda}), F_{\lambda}^{\infty}(u) \geq \frac{1}{N} S_{\lambda}^{\frac{N}{2}}$. Moreover, if $u$ changes sign, then $F_{\lambda}^{\infty}(u) \geq \frac{2}{N} S_{\lambda}^{\frac{N}{2}}$. By Theorem 2.1,

$$
c+o(1)=E_{\lambda, 0}\left(u_{m}\right) \rightarrow E_{\lambda, 0}\left(u^{0}\right)+\sum_{j=1}^{l} F_{0}^{\infty}\left(v_{0}^{j}\right)+\sum_{j=1}^{k} F_{\lambda}^{\infty}\left(v_{1}^{j}\right) .
$$

Suppose that $u^{0} \equiv 0$; then $c=\sum_{j=1}^{l} F_{0}^{\infty}\left(v_{0}^{j}\right)+\sum_{j=1}^{k} F_{\lambda}^{\infty}\left(v_{1}^{j}\right)$. If there exist some $l$ or $k$ such that $v_{0}^{l}$ or $v_{1}^{k}$ changes sign, then by Remark 1.1 (ii) we get $c \geq \frac{2}{N} S_{\lambda}^{\frac{N}{2}}$, which is a contradiction, so we can assume $v_{0}^{i} \geq 0, v_{1}^{j} \geq 0(1 \leq i \leq l, 1 \leq j \leq k)$. By the uniqueness of solutions of problem $\left(P_{0}^{\infty}\right)$ and $\left(P_{\lambda}^{\infty}\right)$ (see Remark 1.1 (i)), we have $c=\frac{l}{N} S_{0}^{\frac{N}{2}}+\frac{k}{N} S_{\lambda}^{\frac{N}{2}}$, which is impossible. So $u^{0} \not \equiv 0$, and we can infer $E_{\lambda, 0}\left(u^{0}\right) \geq \frac{1}{N} S_{\lambda}^{\frac{N}{2}}$, which follows that $l=0, k=0$. Hence $u_{m} \rightarrow u^{0}$ in $H_{0}^{1}(\Omega)$.

Remark 2.6. (i) Let

$$
\begin{aligned}
& \sigma=\left\{E_{\lambda, \epsilon}(u) \mid u \in H_{0}^{1}(\Omega) \text { solves }\left(P_{\lambda}\right)\right\}, \\
& \sigma_{0}=\left\{F_{0}^{\infty}(u) \mid u \in H_{0}^{1}\left(R^{N}\right) \text { solves }\left(P_{0}^{\infty}\right)\right\} \\
& \sigma_{\lambda}=\left\{F_{\lambda}^{\infty}(u) \mid u \in H_{0}^{1}\left(R^{N}\right) \text { solves }\left(P_{\lambda}^{\infty}\right)\right\}
\end{aligned}
$$

be the "spectral" of $\left(P_{\lambda}\right),\left(P_{0}^{\infty}\right)$ and $\left(P_{\lambda}^{\infty}\right)$, respectively. Then in particular, Theorem 2.1 implies that any sequence $\left\{u_{m}\right\}$ satisfying $E_{\lambda, \epsilon}\left(u_{m}\right) \rightarrow \beta, D E_{\lambda, \epsilon}\left(u_{m}\right) \rightarrow 0$ in $H^{-1}(\Omega)(m \rightarrow \infty)$ is strongly relatively compact in $H_{0}^{1}(\Omega)$, provided

$$
\beta \bar{\in}\left\{\bar{\beta}+\sum_{i=1}^{k} \beta_{0}^{i}+\sum_{i=1}^{l} \beta_{\lambda}^{i} \mid \bar{\beta} \in \sigma \backslash \beta, \beta_{0}^{i} \in \sigma_{0} \backslash 0, \beta_{\lambda}^{i} \in \sigma_{\lambda} \backslash 0\right\} .
$$

(ii) Assume $u_{\epsilon} \in H_{0}^{1}(\Omega)$ is the ground state solution of $\left(P_{\lambda, \epsilon}\right), 0<\lambda<\bar{\lambda}-1$. Then $\left|\nabla u_{\epsilon}\right|^{2}-\frac{\lambda u_{\epsilon}^{2}}{|x|^{2}} \rightarrow S_{\lambda} \delta_{0}$ in the sense of measure as $\epsilon \rightarrow 0$, where $\delta_{x}$ denotes the Dirac mass at $x$.

\section{ACKNOWLEDGEMENT}

The authors are grateful to Dr. Didier Smets for helpful discussion. 


\section{REFERENCES}

1. Adimurthi and M. Struwe, Global compactness properties of semilinear elliptic equations with critical exponential growth, J. Funct. Anal., 175(2000), 125-167. MR 2001g:35063

2. H.Brezis and E.Lieb, A relation between pointwise convergence of functions and convergence of functionals, Proc. AMS., 88(1983),486-490. MR 84e:28003

3. H. Brezis and L. Nirenberg, Positive solutions of nonlinear elliptic equations involving critical Sobolev exponent, Comm. Pure Appl. Math., 36(1983), 437-477. MR 84h:35059

4. F. Catrina and Z.Q. Wang, On the Caffarelli-Kohn-Nirenberg inequalities:sharp constants, existence(and nonexistence), and symmetry of extremal functions, Comm. Pure Appl. Math., 53(2000), 1-30.

5. K.S.Chou and C.W. Chu, On the best constant for a weighted Sobolev-Hardy inequality, J. London Math. Soc., 48(1993), 137-151. MR 94h:46052

6. J. M. Coron, Topologie et cas limite des injections de Sobolev, C. R. Acad. Sci. Paris, Ser.I, 299(1984), 209-212. MR 86b:35059

7. W.-Y. Ding, Positive solution of $\Delta u+u^{\frac{N+2}{N-2}}=0$ on contractible domains, J. Partial Diff. Equats., 2(1989), 83-88. MR 91a:35012

8. H. Egnell, Elliptic boundary value problems with singular coefficients and critical nonlinearities, Indiana Univ. Math. J., 38(1989), 235-251. MR 90g:35055

9. J. P. Garcia Azorero and I. Peral Alonso, Hardy inequalities and some critical elliptic and parabolic problems, J. Diff. Equats., 144(1998), 441-476. MR 99f:35099

10. D. Gilbarg and N. S. Trudinger, Elliptic partial differential equations of second order, Second edition, Spring-Verlag, Berlin-Heidelberg-New York-Tokyo, 1983. MR 86c:35035

11. E. Jannelli, The role played by space dimension in elliptic critical problems, J. Diff. Equats., 156(1999), 407-426. MR 2000f:35053

12. P. L. Lions, The concentration compactness principle in the calculus of variations. The limit case (I), Revista Math. Ibero., 1(1985), 145-201. MR 87c:49007

13. D. Pierotti and S. Terracini, On a Neumann problem with critical exponent and critical nonlinearity on the boundary, Comm. PDE, 20(1995), 1155-1187. MR 96f:35060

14. M. Struwe, A global compactness result for elliptic boundary value problems involving limiting nonlinearities, Math. Z., 187(1984), 511-517. MR 86k:35046

15. S. Terracini, On positive solutions to a class equations with a singular coefficient and critical exponent, Adv. Diff. Equats., 2(1996), 241-264. MR 97b:35057

16. S.Yan, A global compactness result for quasilinear elliptic boundary value problems involving limiting nonlinearities, Chinese Ann. Math., 16A(1995), 397-402.

Institute of Applied Mathematics, Academy of Mathematics and System Sciences, Chinese Academy of Sciences, Beijing 100080, People's Republic of China

E-mail address: dmcao@mail.amt.ac.cn

Department of Mathematics, Xiao Gan University, Xiao Gan, People's Republic of China - And - Institute of Applied Mathematics, AMSS., Chinese Academy of Sciences, Beijing 100080, People's Republic of China

E-mail address: pengsj@mail.amss.ac.cn 